\title{
Evolving treatment paradigms for PCV
}

\author{
Beau J. Fenner ${ }^{1,2}$, Chui Ming Gemmy Cheung ${ }^{1,2}$, Shaun S. Sim $\mathbb{D}^{1}{ }^{1}$, Won Ki Lee ${ }^{3}$, Giovanni Staurenghi ${ }^{4}$, Timothy Y. Y. Lai $\mathbb{D}^{5}$, \\ Paisan Ruamviboonsuk ${ }^{6}$, Gregg Kokame ${ }^{7}$, Yasuo Yanagi ${ }^{8}$ and Kelvin Y. C. Teo $(\mathbb{D})^{1,2,9}$
}

(c) The Author(s) 2021

Polypoidal choroidal vasculopathy (PCV) is a subtype of neovascular AMD ( $\mathrm{AMD}$ ) that accounts for a significant proportion of nAMD cases worldwide, and particularly in Asia. Contemporary PCV treatment strategies have closely followed those used in typical $\mathrm{nAMD}$, though there are significant gaps in knowledge on PCV management and it remains unclear if these strategies are appropriate. Current clinical trial data suggest intravitreal anti-vascular endothelial growth factor (VEGF) therapy alone or in combination with photodynamic therapy is effective in managing haemorrhage and exudation in PCV, although the optimal treatment interval, including as-needed and treat-and-extend approaches, is unclear. Newer imaging modalities, including OCT angiography and high-resolution spectral domain OCT have enabled characterisation of unique PCV biomarkers that may provide guidance on how and when treatment and re-treatment should be initiated. Treatment burden for PCV is a major focus of future therapeutic research and several newly developed anti-VEGF agents, including brolucizumab, faricimab, and new modes of drug delivery like the port delivery system, offer hope for dramatically reduced treatment burden for PCV patients. Beyond anti-VEGF therapy, recent developments in our understanding of PCV pathophysiology, in particular the role of choroidal anatomy and lipid mediators in PCV pathogenesis, offer new treatment avenues that may become clinically relevant in the future. This article explores the current management of PCV and more recent approaches to PCV treatment based on an improved understanding of this unique disease process.

Eye (2022) 36:257-265; https://doi.org/10.1038/s41433-021-01688-7

\section{INTRODUCTION}

Age-related macular degeneration (AMD) is a leading cause of irreversible vision loss worldwide [1, 2]. Several subtypes of neovascular AMD (nAMD) can be characterised based on multimodal imaging [3]. Polypoidal choroidal vasculopathy (PCV), while currently considered a subtype of nAMD, is more common in Asian populations, comprising up to $50 \%$ of nAMD cases presenting to retina specialist clinics [4-7]. Morphologically, the lesion complex in PCV consists of polypoidal lesions (PL) with an associated branching neovascular network (BNN) best detected using indocyanine green angiography (ICGA) [8-10]. In addition to clinical features, differences in the pathogenesis of PCV and typical nAMD have also been proposed. For example, drusen and pigmentary changes which are considered the hallmark of early AMD are not commonly seen in PCV. In contrast, a congested choroidal background and association with a history of central serous chorioretinopathy have been reported to be common in PCV.

Despite these differences, current therapeutic options of PCV have largely evolved from treatments designed for typical nAMD. In this review we will discuss how the latest clinical trials in PCV, coupled with improved understanding of pathogenesis from imaging studies, have progressively contributed to the formulation of PCV-specific treatment approaches. We go on to highlight some emerging treatments that may soon play an important role in PCV management.

\section{CURRENT PCV TREATMENT OPTIONS}

Prior to the availability of optical coherence tomography, the $\mathrm{PL}$ was the main target of treatment, as this was the most obvious source of haemorrhage and exudation based on clinical examination, fluorescein angiography, and ICGA. Closure of the PL with focal laser or subsequently with verteporfin photodynamic therapy (PDT) have been widely used [11-15].

Historically, the management of PCV with PDT was complicated by acute vision loss and massive subretinal haemorrhage [16]. These complications were reduced with the introduction of antivascular endothelial growth factor (VEGF) therapies. The EVEREST trial [17] was one of the earliest PCV-specific randomised controlled trials. The sample size was limited and the primary endpoint was based on PL closure rate assessed with ICGA. This trial showed that PDT was superior to ranibizumab monotherapy in achieving PL closure (78\% versus $29 \%$ after 6 months). With the results from the EVEREST study, PDT remained popular as the first choice of treatment in PCV. Subsequently, the LAPTOP trial [18]

\footnotetext{
${ }^{1}$ Singapore National Eye Centre and Singapore Eye Research Institute, Singapore, Singapore. ${ }^{2}$ Duke-NUS Graduate Medical School, National University of Singapore, Singapore,

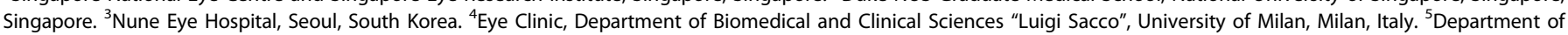
Ophthalmology and Visual Sciences, The Chinese University of Hong Kong, Hong Kong Eye Hospital, Hong Kong, China. ${ }^{6}$ Department of Ophthalmology, Rajavithi Hospital, Bangkok, Thailand. ${ }^{7}$ Division of Ophthalmology, Department of Surgery, University of Hawaii School of Medicine, Honolulu, HI, USA. ${ }^{8}$ Department of Ophthalmology and Microtechnology, Yokohama City University, Yokohama, Japan. ${ }^{9}$ University of Sydney, Sydney, Australia. ${ }^{凶}$ email: kelvin.teo.y.c@singhealth.com.sg
}

Received: 31 May 2021 Revised: 28 June 2021 Accepted: 1 July 2021

Published online: 14 July 2021 

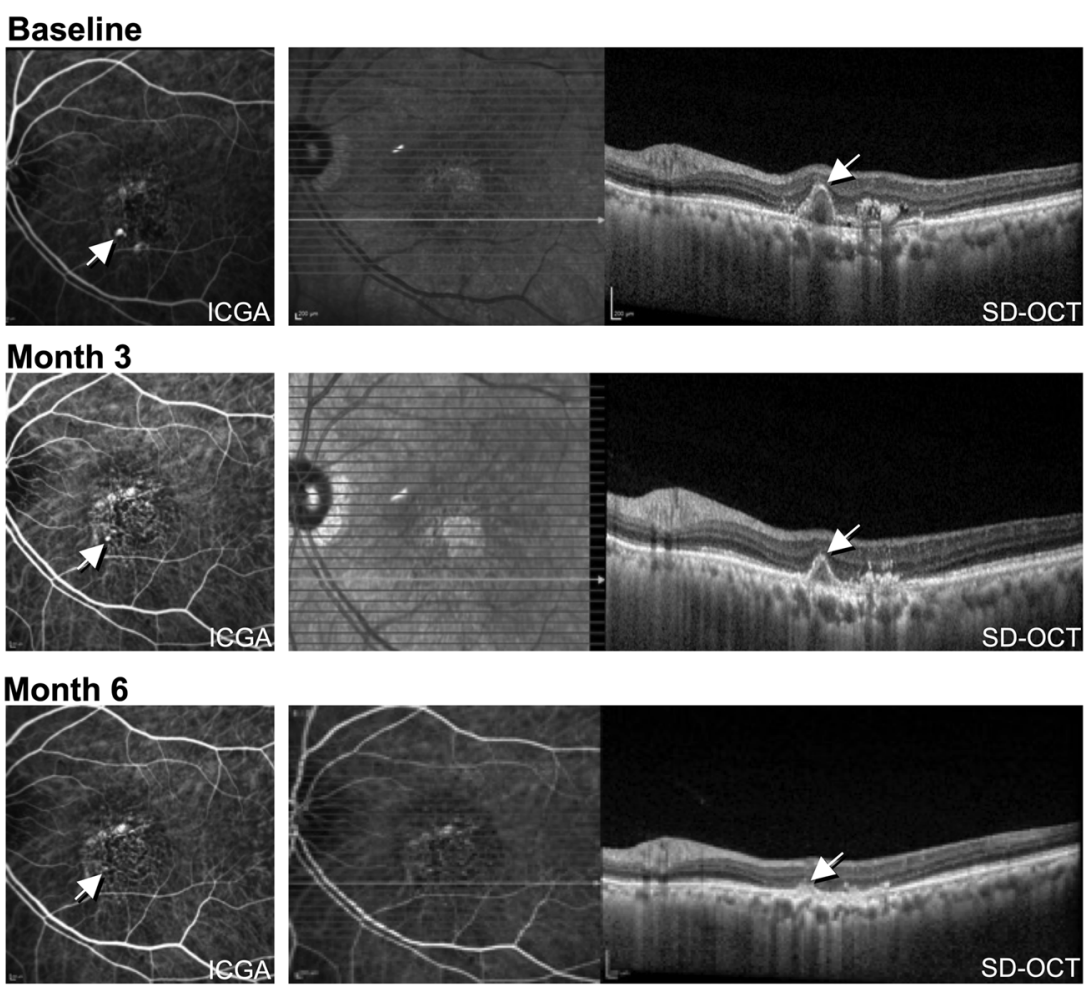

Fig. 1 Demonstration of polyp closure in PCV following treatment with intravitreal aflibercept. Images were obtained using indocyanine green angiography (ICGA, shown on left) and spectral domain optical coherence tomography (SD-OCT, shown on right) at baseline, 3 , and 6 months following treatment initiation. The polypoid lesion is indicated with an arrow, with progressive closure seen at 3 and 6 months.

compared PDT monotherapy with ranibizumab monotherapy in PCV and reported superior visual outcome in the ranibizumab arm. However, PL closure rate was not reported and this has been criticised by some as a weakness of the study at the time of reporting [19]. However, the findings from the LAPTOP trial did serve to confirm a prevailing hypothesis that good functional outcomes are achievable even without PDT, and that PL closure may not necessarily be related to functional outcomes. In the EVEREST II study [20], ranibizumab combined with PDT achieved superior visual gains compared to ranibizumab alone (8.3 versus 5.1 ETDRS letters, at 12 months) while at the same time also achieving a higher rate of PL closure (69.3\% versus $34.7 \%)$. These studies suggested a benefit of combining anti-VEGF therapy with PDT and has led to a decline in PDT monotherapy.

The PLANET study $[21,22]$ evaluated the efficacy of intravitreal aflibercept in the management of PCV, either as monotherapy or with limited use of rescue PDT for patients unresponsive to aflibercept monotherapy. The primary endpoint demonstrated that the monotherapy arm was non-inferior to the combination arm (10.7 versus 10.8 ETDRS letter gains), although due to the strict criteria for the use of PDT, most of the combination therapy group (about $85 \%$ of patients) completed the study with only antiVEGF monotherapy. In terms of anatomical outcome, most eyes (nearly $80 \%$ ) had a fluid-free retina based on OCT at the end of the study, despite $<40 \%$ of eyes achieving PL closure on ICGA (see Fig. 1). The PLANET study demonstrated the apparent efficacy and safety of anti-VEGF monotherapy in PCV and, in addition, the results suggested that good visual outcomes are compatible with the presence of an unclosed PL. The proposed 'polyp inactivation', characterised by absence of fluid despite PL persistence also initiated further research questioning the significance of $\mathrm{PL}$ closure.

Results of these therapeutic studies were also important in the evolving understanding of the nature of PCV lesions. While earlier studies emphasised the difference in the benefit of PDT in PCV compared to typical nAMD, the two lesions had been proposed to be distinct entities. With more recent studies which demonstrate that anti-VEGF therapy can be used successfully as first-line therapy in PCV and typical nAMD, the similarities of these two subtypes were increasingly appreciated. Concurrently most imaging and histopathology studies place the BNN in PCV lesions in a plane between the retinal pigment epithelium (RPE) and Bruch's membrane. This finding further supports a conclusion that the BNN is a type 1 MNV instead of an intrachoroidal vascular channel as suggested in earlier studies based on ICGA, which did not have the benefit of depth resolution that OCT offers. There have also been some reports that PCV may uncommonly display a type 2 MNV appearance, affirming similar features to the typical nAMD subtype $[23,24]$.

\section{ANTI-VEGF TREATMENT REGIMENS FOR PCV}

Recognising that the BNN component of the PCV lesion is neovascular in nature, the anti-VEGF treatment regimens in PCV have also progressively become more aligned with that in typical nAMD. A popular treatment protocol for typical nAMD is the treat and extend (T\&E) regimen. Briefly, the T\&E protocol dictates that patients receive three initial monthly loading doses of anti-VEGF therapy followed by progressive adjustment of the treatment interval according to anatomical and functional outcomes. This interval can be extended by weeks or months with the aim of reducing treatment burden. These regimens use disease activity markers of fluid in retinal compartments, visual acuity and presence of haemorrhage on clinical examination for retreatment decisions. While not as extensively studied, PCV patients undergoing a T\&E protocol show favourable outcomes comparable to patients with typical nAMD [25-31]. However, the current disease activity indicators used for guiding subsequent retreatment 
decisions are based on OCT features derived from experience in typical nAMD. It remains unclear if similar disease activity markers are sufficient for the optimal treatment of PCV. For example, recent evidence for more "relaxed" retreatment criteria for typical nAMD demonstrated comparable outcomes to a strict "fluid free" strategy. Here, the FLUID study demonstrated that up to $200 \mu \mathrm{m}$ of subretinal fluid (SRF) at the fovea was well-tolerated over 2 years in nAMD cases, although it did not differentiate between nAMD subtypes [32]. However, given the prominence of SRF as a marker of disease activity in PCV, there is clearly a need to determine whether SRF is similarly well-tolerated in PCV. While some clinicians have already adopted the findings of the FLUID study into their practice for certain PCV patients, there is a need to confirm these findings with a controlled clinical trial with PCV patients. More recent work from several groups [33-35] demonstrates that fluctuations in retinal thickness results in poorer visual outcomes, and this finding, combined with the observation from EVEREST II that eyes managed with combination therapy had less persistent SRF [20], suggest that the FLUID study may be less applicable to PCV management.

In addition to fluid, the PL within the sub-RPE vascular network is responsible for much of the exudation in PCV [9]. A potential limitation of current anti-VEGF treatment protocols is the lack of consideration of $\mathrm{PL}$ status. Chaikitmongkol et al. recently demonstrated a $50 \%$ complete polyp closure rate within 2 months following initiation of aflibercept [36]. Thus the speed of PL closure could be an additional consideration in titrating treatment. In a recent randomised controlled trial, eyes with persistent PL after three initial monthly loading injections of aflibercept that were treated with an additional three monthly injections achieved comparable visual gain and higher PL closure rate at month 6 and month 12 [37]. Addition of PDT is another alternative for eyes with residual PL. This was evaluated in the FUJISAN study [38], which showed that intravitreal ranibizumab combined with initial or deferred PDT had similar functional outcomes (8.1 versus 8.8 ETDRS letter gains in initial and deferred PDT arms, respectively, at 1 year). While the initial PDT arm required fewer additional ranibizumab injections compared to deferred PDT, only about half of the population in the deferred PDT arm required adjunct PDT. The outcomes of the above clinical trials are summarised in Table 1, and their practical application is illustrated in the treatment pathway shown in Fig. 2.

\section{ANATOMICAL ENDPOINTS AND PCV SPECIFIC BIOMARKERS}

In current clinical practice the management of PCV following commencement of initial therapy is guided for the most part by visual acuity, the presence of subretinal and intraretinal fluid, and the status of the PL as mentioned above. While these features have informed the outcomes of previous PCV clinical trials, there is a growing body of literature suggesting several other morphological and physiological features specific to PCV may also have value as treatment biomarkers.

\section{Polypoidal lesions as biomarkers for treatment}

A sharp-peaked PED and a sub-RPE ring-like lesion on OCT have been reported to be frequently associated with and co-localise to PL on ICGA. The Asia Pacific Ophthalmic Imaging Society (APOIS) PCV workgroup has shown that OCT is a viable alternative to ICGA in assessing the presence of PL in treatment naïve eyes as well as in eyes with persistent fluid following monthly anti-VEGF loading treatment [39]. (Fig. 1) Features which suggest presence of PL in eyes with persistent fluid posttreatment include presence of a sharp-peaked PED, a sub-RPE ring on OCT and an orange nodule on colour fundus photography. In addition, the height of the PED as well as reflectivity of the content of the PED may predict PL closure (Tan
A.C. et al., manuscript in review). These OCT features can facilitate the assessment of PL perfusion status especially after treatment with less reliance on ICGA. The APOIS PCV workgroup also demonstrated that if deferred PDT is indicated in eyes with persistent fluid, the appropriate PDT spot size and location can be deduced with excellent accuracy using only OCT coupled with near-infrared [40] (100\% PL coverage and 90\% BNN coverage). Re-examining the baseline scans for an inverted U-shaped elevation in anti-VEGF resistant cases has also been reported to be helpful for detecting PL which might have been overlooked [41].

OCT angiography (OCTA), a novel imaging modality which detects blood flow, may also be able to detect flow signal within the PCV lesion $[42,43]$. Specifically, detection of flow within the BNN has been shown to be comparable or even superior to ICGA due to the high contrast available with OCT. However, ICGA may be superior to OCTA for PL identification due to slow PL filling that is not well seen with OCTA. This imaging finding is consistent with histopathological studies which reported that $\mathrm{PL}$ are aneurysmal dilatations of neovascular tissue [44], and that the $\mathrm{PL}$ arises from the neovascular complex rather than as a primary lesion. The response to anti-VEGF therapy demonstrated in clinical trials discussed in the previous section are compatible with these imaging and histopathological findings $[18,45]$. In addition, the nature of the PL based on OCTA features has been debated. While some investigators describe aneurysmal dilatation in the $\mathrm{PL}$, others have suggested the appearance represented tangled vascular structures [44].

\section{Choroidal features}

PCV resides within the pachychoroid spectrum of diseases. These eyes are characterised by a thickened choroid, attenuation of the choriocapillaris and dilated Haller's layer vessels $[46,47]$. Several additional metrics have been used to quantify and grade the characteristics of the choroid; (1) choroidal thickness, (2) choroidal vascular hyperpermeability $(\mathrm{CVH})$, and (3) choroidal vascularity index (CVI). The association of these choroid features with clinical characteristics and treatment outcomes of PCV is currently inconsistent. Moreover, thickening of the choroid is often seen in normal eyes and while it may be more common among PCV patients, it may not form an intrinsic part of PCV pathogenesis.

A thicker choroid was shown to be associated with a poorer anatomical response to initial loading doses of anti-VEGF [48]. Recent work by Chang and Cheng [49] has determined a subfoveal choroidal thickness (SFCT) of $267.5 \mu \mathrm{m}$ as a useful threshold. Higher SFCT is more than $50 \%$ less likely to achieve resolution of SRF within three months of anti-VEGF initiation when compared to normal choroid patients. Choroidal thickness is also positively correlated to PCV lesion area [50] and presumably related to treatment outcomes. On the other hand, a post hoc analysis of imaging biomarkers of the EVEREST II cohort did not show any association between central choroidal thickness and functional or anatomical outcomes [51]. Similarly, CVH and CVI association with treatment outcomes is variable. Some described the favourable outcomes with the presence of $\mathrm{CVH}$ with lower number of anti-VEGF treatments combined with PDT [52], while other studies showed that the presence of CVH was associated with poor visual and anatomical outcomes after treatment with anti-VEGF $[53,54]$.

\section{Pigment epithelial detachment}

Pigment epithelial detachments (PEDs) are a hallmark feature of PCV $[55,56]$. PEDs in PCV patients tend to be larger than those in typical nAMD and are associated with a higher incidence of RPE tears but their morphology is not prognostic of visual outcomes at one year [56]. In addition, PEDs, when analysed as a biomarker in any $\mathrm{nAMD}$, were found to be compatible with good 
은

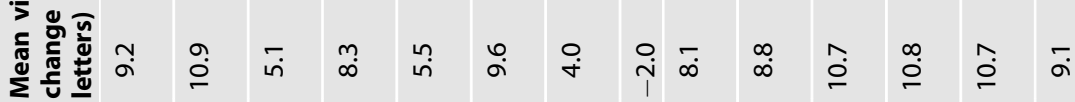

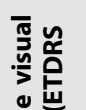

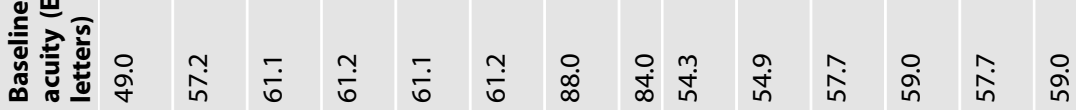

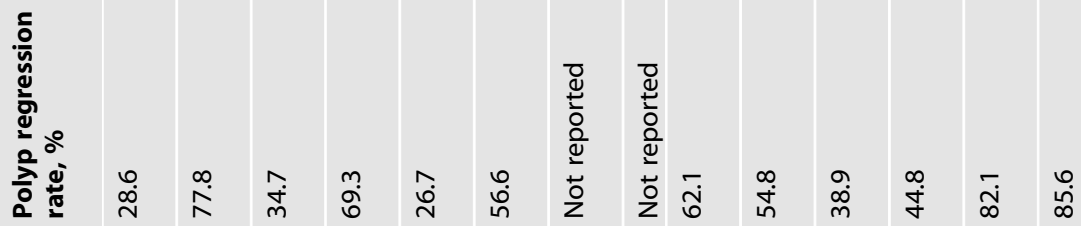

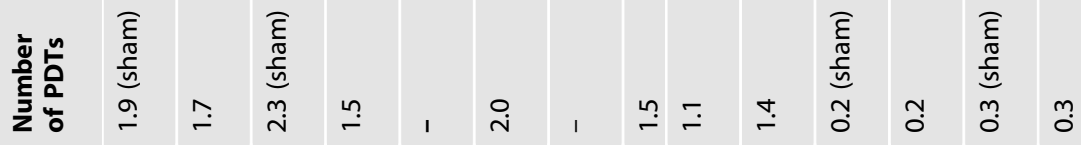

$\stackrel{\dot{\infty}}{\stackrel{\circ}{\circ}}$

至苛

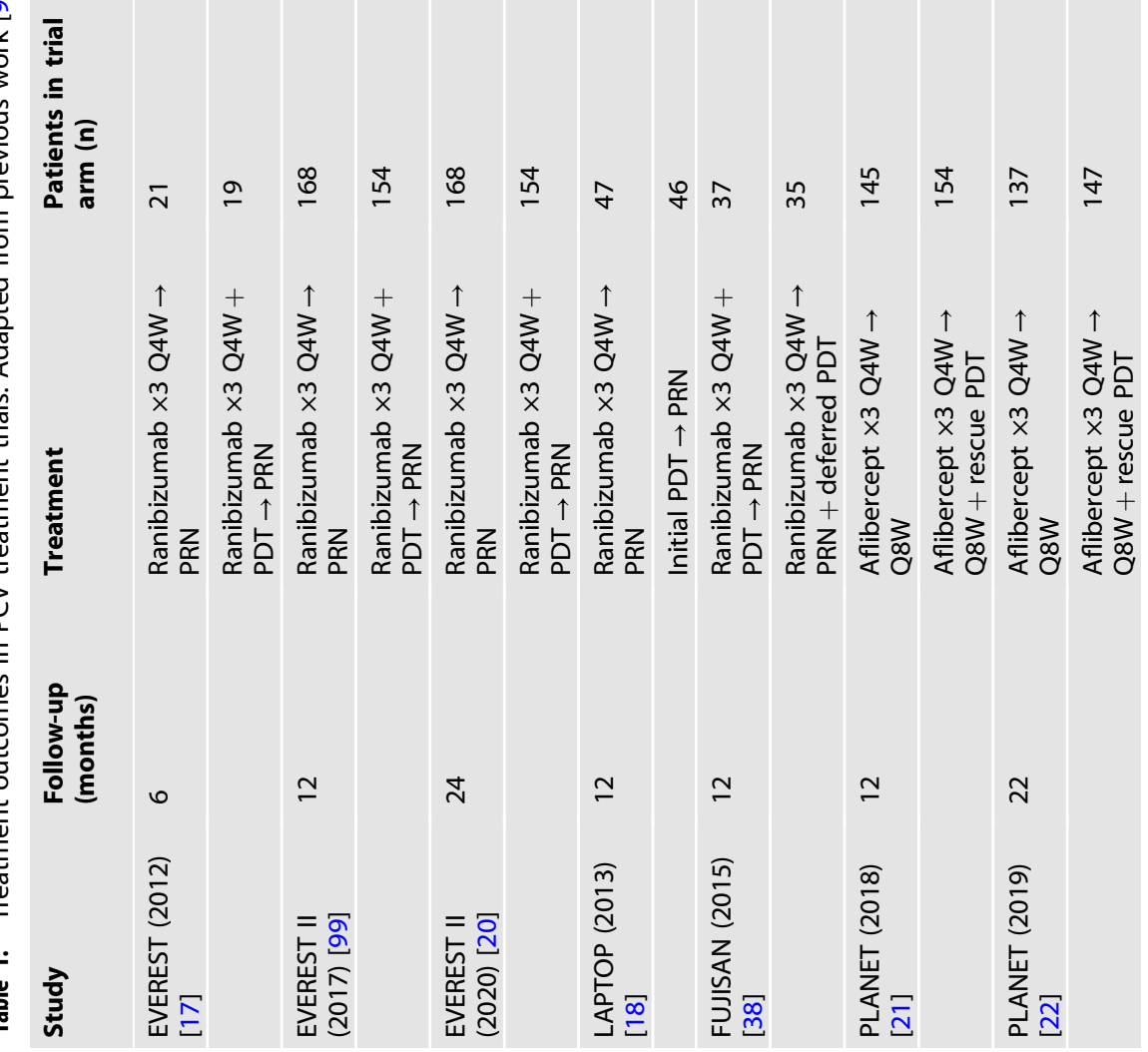




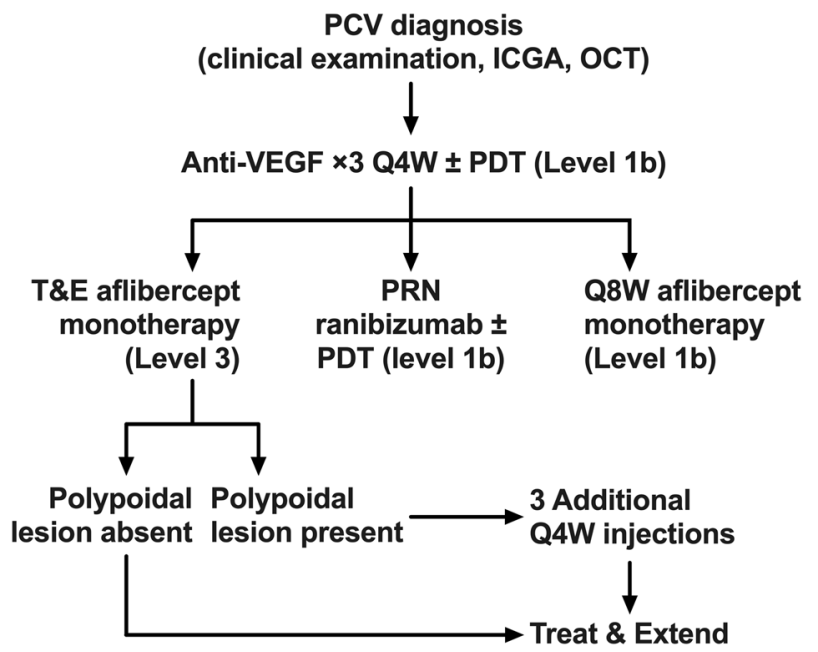

Fig. 2 Treatment pathways for PCV. Anti-VEGF monotherapy can be used based on outcomes from the PLANET trial $[21,22]$, or combination therapy used based on outcomes from the EVEREST trials $[17,20,99]$. Alternatively, recent work suggests that PCV may be treated with potentially fewer injections and better functional outcomes using a treat-and-extend approach.

vision and did not have a significant impact on visual acuity with treatment [56].

Novel metrics for quantification for PCV-specific biomarkers Currently, neither choroidal thickness nor PED have been consistently shown to be associated with outcomes in patients treated for PCV. Most studies have used subfoveal or central choroidal thickness and maximum height or width of PED, or merely the presence or absence of PED. These point measurements and binary grading criteria may not fully encompass the effects of these features on the disease process or activity. With recent advances in segmentation algorithms, volumetric analysis of retinal pathological features such as IRF and SRF have been suggested as useful biomarkers for predicting treatment response $[34,57,58]$. When similarly applied to PED and the choroid, volumetric analysis may provide much more information regarding the impact of these features on the outcomes of PCV. In a post hoc analysis of a PCV monotherapy treatment trial we performed, we found that baseline PED volume, lower CVI and a more rapid reduction in PED volume from baseline to month 3 are associated with reduced disease activity at month 12 . These features have yet to be validated as retreatment criteria but show promise in this preliminary work [37].

\section{NOVEL THERAPIES ON THE HORIZON FOR PCV}

One of the core goals of treatment for neovascular maculopathies is to minimise treatment burden for the patient. There has been considerable effort in the field to either modify the delivery of currently available drugs through less intensive regimens or develop new drugs with the aim of improving treatment durability, efficacy, or safety, compared to currently available agents. While most ongoing registration clinical trials are aimed at nAMD, it is likely that in these cohorts, a subset are $P C V$. Increasingly, there is interest in investigating the efficacy of these newer agents on PCV.

Brolucizumab is a recently available antibody fragment developed for the management of nAMD and other exudative maculopathies and was found to be similarly efficacious but more durable than aflibercept in the phase 3 HAWK and HARRIER trials $[59,60]$. Subgroup analysis of Japanese PCV patients from the HAWK trial showed excellent anatomical and functional outcomes with 8- or 12weekly injections compared to 8-weekly injections with aflibercept [61]. A recent report by Matsumoto et al. [62] on the 3-month outcomes of type 1 CNV patients (including 19 with PCV) treated with three loading doses of brolucizumab showed polyp regression in $78.9 \%$ of cases, combined with impressive visual and anatomical outcomes. Despite these promising findings, the adoption of brolucizumab by retinal physicians had been hampered by reports of intraocular inflammation, in particular occlusive vasculitis, which has in some cases led to permanent visual loss [63].

Another upcoming antibody for nAMD treatment, faricimab, is a bi-specific monoclonal antibody that binds VEGF-A and angiopoietin-2 (Ang-2), the latter being up-regulated in neovascular membranes and promoting vascular leakage in combination with VEGF-A [64, 65]. Recently released data from the phase 3 TENAYA and LUCERNE studies [66] have demonstrated that faricimab is non-inferior to aflibercept in terms of functional and structural outcomes in nAMD patients, but can be used with longer treatment intervals of up to 16 weeks, compared to every 8 weeks for patients treated with aflibercept. There is no report of the efficacy and safety of faricimab on PCV yet. The additional blockage of Ang-2 has been proposed to promote vascular stability. Whether this translates into any significant anatomical advantage in typical $\mathrm{nAMD}$ or PCV remains to be seen.

An alternative to regular intravitreal injections for long-term nAMD treatment is the surgically implanted port delivery system (PDS) for ranibizumab [67]. The PDS is a small refillable reservoir for an anti-VEGF antibody that is surgically implanted at the pars plana via scleral cutdown and can be periodically refilled via direct injection through the conjunctiva. A PDS loaded with $100 \mathrm{mg} / \mathrm{mL}$ ranibizumab had a median reload time of 15 months but with comparable efficacy to monthly ranibizumab injections in the phase 2 LADDER trial [67]. The real-world safety profile of the PDS remains to be seen, but at present it shows promise for patients in need of long-term anti-VEGF therapy who wish to avoid frequent injections. There is no study of the PDS in eyes with PCV to-date.

\section{FUTURE RESEARCH DIRECTIONS Developing PCV-specific biomarkers}

Heterogeneity among PCV lesion characteristics and treatment responses is well recognised. In EVEREST II better visual gains were achieved, somewhat predictably, among those with younger age, poorer baseline vision, and smaller PCV lesion size for patients in the ranibizumab monotherapy arm [51]. Higher vision gains are associated with higher anti-VEGF injection frequency [4], CVH [52], and thinner subfoveal choroid [48], while poorer visual outcomes have been reported in cases with fibrovascular PEDs [68] or subretinal fibrosis [69], and sub macular haemorrhage [70]. Furthermore, in the EVEREST II study, a subgroup of patients $(\sim 30 \%)$ in the combination arm required only 3-4 intravitreal ranibizumab injections over 2 years [20]. However, there is currently no reliable method to prospectively identify this subgroup. Advanced image analysis tools and characterization of PCV-specific biomarkers beyond those recognised in typical nAMD will further develop personalisation of PCV therapies.

\section{Understand phenotypes associated with poor vision outcome- fibrosis and macular atrophy}

The end-stage phenotypes of nAMD are well described and can represent a stage of disease in which treatment is often futile. Both fibrosis and macular atrophy have been associated with various baseline clinical and treatment features such as nAMD subtype, visual acuity and frequency of treatment. [45, 71-75] These phenotypes, however, are not well understood in the PCV subtype. Incident fibrosis was studied in an Asian population with about $48 \%$ of the cohort identified as having PCV [69]. There was a threefold increase in incident fibrosis (from 10\% noted at baseline 
to about $30 \%$ at 12 months), but no difference in the rate of subretinal fibrosis between PCV and typical nAMD. The rate of macular atrophy was found to be lower than prior reports. Taken together, these findings suggest that fibrosis was a more significant end stage phenotype than macular atrophy in Asian (PCV) patients [69]. Further understanding of the developmental differences of fibrosis between PCV and typical nAMD, as well as molecular pathways involved in the development of fibrosis, are necessary and offer opportunities for targeted therapies against these phenotypes.

\section{Long-term recurrence}

In typical nAMD, reports of up to 10-year outcomes are available $[76,77]$. In contrast, currently available clinical trial data for PCV mostly report visual improvements in the range of 5-10 letter gain at 2 years [20, 22]. A small number of real-world retrospective reports on the 5-year outcomes for PCV patients suggest that visual outcomes and treatment burdens are similar to typical nAMD [78]. In PCV eyes, however, there appears to be a distinct period of lesion reactivation that occurs at around 2-3 years after initial presentation that requires reversion to a more intensive treatment regimen [79]. Previous work from Kim et al. [80] suggests that the presence of clusters of multiple PL and larger lesion size are associated with PCV reactivation during the first year, though it is unclear what features dictate reactivation in the long-term and such data would be valuable when formulating management strategies for patients [81]. In addition, data on longterm incidence of submacular haemorrhage in PCV is lacking. A recent study found that submacular haemorrhage occurred in $10 \%$ in 5 years and $30 \%$ at 10 years. This rate is significantly higher than that of typical nAMD, reported to occur in only $4 \%$ in 5 years and $10 \%$ in 10 years [82]. The presence of a cluster of polyps was found to be significantly associated with submacular haemorrhage, further supporting the importance of PL status as a treatment endpoint for PCV.

\section{Addressing choroidal background}

Therapies that directly address choroidal congestion may be effective for PCV treatment due to the strong evidence for choroidal features playing a role in the pathophysiology of PCV. Currently, PDT offers the most potential as a therapeutic option that targets the choroid. Some studies have demonstrated a reduction in CVH and choroidal thickness after PDT [83-85]. Further evidence of the effects of PDT on the choroid has been proposed in the form of modulation of large choroidal vessels in the Haller's layer. However, this effect appears short-lived and the dilated vessels return to their original configuration eventually [86]. Taken together, these observations suggest that current therapies reduce the leakiness of the choroid but effects may be transient as the dilated vessels may be an irreversible feature resulting from chronic structural remodelling and the formation of anastomoses $[87,88]$. These features may not be easily reversed by pharmacological means and further research into their mechanism of formation is required.

\section{Upstream mediators in PCV pathogenesis}

Most current PCV treatment strategies still hinge on the role of VEGF as a mediator of choroidal neovascularization and vascular permeability, a role which has been appreciated for 25 years [89]. More recent work has focused on other physiological mediators that may play a role in PCV pathogenesis. Work by Jones et al. [90] and Kumar et al. [91] showed that the serine protease HtrA1, when overexpressed in transgenic mice, was sufficient to induce cardinal features of PCV including a branching network of choroidal vessels and $P L$ via a mechanism involving thinning of Bruch's membrane and VEGF stimulation. Several components of the lipid metabolome have been linked to PCV through epidemiologic, genetic and biochemical studies [92-94]. Metabolomic studies have implicated several oxidised lipids and amino acids, including hyodeoxycholic acid and L-tryptophanamide, as retinal epithelial modulators with differential abundance in PCV compared to typical nAMD [95]. Both species influenced apoptosis and necrosis in cultured RPE and retinal endothelial cells. A synergy between oxidised lipids and $\mathrm{HtrA} 1$ to promote VEGF expression and ultimately stimulate neovascular membrane development was recently appreciated [96]. Anti-HtrA1 antibodies are already under investigation for management of geographic atrophy [97], though it remains to be seen if this class of drugs also have utility in the treatment of PCV.

\section{CONCLUSIONS}

This review demonstrates how the understanding of PCV has evolved with imaging studies and therapeutic trials. These advances have in turn translated into treatments, which promote sustained functional outcome and are aligned with the pathophysiology of the condition. There remains significant heterogeneity among retinal physicians in terms of preferred PCV treatment approaches, although clinical outcomes have improved substantially since the initial discovery of PCV. Recurrence of the disease 2-3 years after successful treatment is still a major concern. Future work which addresses the unique aspects of this condition will no doubt provide additional treatment options specifically tailored for PCV in the form of expanded drug choice and alternative dosing regimens, likely with a strong focus on improved treatment durability and reduced treatment burden.

\section{REFERENCES}

1. Jager RD, Mieler WF, Miller JW. Age-related macular degeneration. N Engl J Med. 2008;358:2606-17.

2. Lim LS, Mitchell P, Seddon JM, Holz FG, Wong TY. Age-related macular degeneration. Lancet. 2012;379:1728-38.

3. Spaide RF, Jaffe GJ, Sarraf D, Freund KB, Sadda SR, Staurenghi G, et al. Consensus Nomenclature for Reporting Neovascular Age-Related Macular Degeneration Data: consensus on Neovascular Age-Related Macular Degeneration Nomenclature Study Group. Ophthalmology. 2020;127:616-36.

4. Fenner BJ, Ting DSW, Tan ACS, Teo K, Chan CM, Mathur R, et al. Real-World Treatment Outcomes of Age-Related Macular Degeneration and Polypoidal Choroidal Vasculopathy in Asians. Ophthalmol Retin. 2020;4:403-14.

5. Lorentzen TD, Subhi Y, Sørensen TL. Prevalence of polypoidal choroidal vasculopathy in white patients with exudative age-related macular degeneration: systematic review and meta-analysis. Retina. 2018;38:2363-71.

6. Fujiwara K, Yasuda M, Hata J, Oshima Y, Hashimoto S, Yoshitomi T, et al. Prevalence and Risk Factors for Polypoidal Choroidal Vasculopathy in a General Japanese Population: the Hisayama Study. Semin Ophthalmol. 2018;33:813-9.

7. Liew G, Hyun-Jin HD, Hooper C, Chia EM, Mitchell P, Ong S, et al. Prevalence of polypoidal choroidal vasculopathy in Caucasian patients as estimated from optical coherence tomography signs. Eye (Lond). 2021;35:1011-2.

8. Yannuzzi LA, Sorenson J, Spaide RF, Lipson B. Idiopathic polypoidal choroidal vasculopathy (IPCV). Retina. 1990;10:1-8.

9. Cheung CMG, Lai TYY, Ruamviboonsuk P, Chen SJ, Chen Y, Freund KB, et al. Polypoidal choroidal vasculopathy: definition, pathogenesis, diagnosis, and management. Ophthalmology. 2018;125:708-24.

10. Ciardella AP, Donsoff IM, Huang SJ, Costa DL, Yannuzzi LA. Polypoidal choroidal vasculopathy. Surv Ophthalmol. 2004;49:25-37.

11. Spaide RF, Donsoff I, Lam DL, Yannuzzi LA, Jampol LM, Slakter J, et al. Treatment of polypoidal choroidal vasculopathy with photodynamic therapy. Retina. 2002;22:529-35.

12. Quaranta M, Mauget-Faÿsse M, Coscas G. Exudative idiopathic polypoidal choroidal vasculopathy and photodynamic therapy with verteporfin. Am J Ophthalmol. 2002;134:277-80.

13. Chan WM, Lam DS, Lai TY, Liu DT, Li KK, Yao Y, et al. Photodynamic therapy with verteporfin for symptomatic polypoidal choroidal vasculopathy: one-year results of a prospective case series. Ophthalmology. 2004;111:1576-84.

14. Rogers AH, Greenberg PB, Martidis A, Puliafito CA. Photodynamic therapy of polypoidal choroidal vasculopathy. Ophthalmic Surg Lasers Imaging. 2003; 34:60-3.

15. Lee SC, Seong YS, Kim SS, Koh HJ, Kwon OW. Photodynamic therapy with verteporfin for polypoidal choroidal vasculopathy of the macula. Ophthalmologica. 2004;218:193-201. 
16. Gomi F, Sawa M, Wakabayashi T, Sasamoto Y, Suzuki M, Tsujikawa M. Efficacy of intravitreal bevacizumab combined with photodynamic therapy for polypoidal choroidal vasculopathy. Am J Ophthalmol. 2010;150:48-54.e41.

17. Koh A, Lee WK, Chen LJ, Chen SJ, Hashad Y, Kim H, et al. EVEREST study: efficacy and safety of verteporfin photodynamic therapy in combination with ranibizumab or alone versus ranibizumab monotherapy in patients with symptomatic macular polypoidal choroidal vasculopathy. Retina. 2012;32:1453-64.

18. Oishi A, Kojima H, Mandai M, Honda S, Matsuoka T, Oh H, et al. Comparison of the effect of ranibizumab and verteporfin for polypoidal choroidal vasculopathy: 12 month LAPTOP study results. Am J Ophthalmol. 2013;156:644-51.

19. Tan CS, Ngo WK, Lim LW. Re: Oishi et al.: LAPTOP study: a 24-month trial of verteporfin versus ranibizumab for polypoidal choroidal vasculopathy (Ophthalmology 2014;121:1151-2). Ophthalmology. 2015;122:e5-6.

20. Lim TH, Lai TYY, Takahashi K, Wong TY, Chen LJ, Ruamviboonsuk P, et al. Comparison of Ranibizumab With or Without Verteporfin Photodynamic Therapy for Polypoidal Choroidal Vasculopathy: the EVEREST II Randomized Clinical Trial. JAMA Ophthalmol. 2020;138:935-42.

21. Lee WK, lida T, Ogura Y, Chen SJ, Wong TY, Mitchell P, et al. Efficacy and Safety of Intravitreal Aflibercept for Polypoidal Choroidal Vasculopathy in the PLANET Study: a randomized clinical trial. JAMA Ophthalmol. 2018;136:786-93.

22. Wong TY, Ogura Y, Lee WK, lida T, Chen SJ, Mitchell P, et al. Efficacy and Safety of Intravitreal Aflibercept for Polypoidal Choroidal Vasculopathy: 2-Year Results of the PLANET Study. Am J Ophthalmol. 2019;204:80-9.

23. Kokame GT. Prospective evaluation of subretinal vessel location in polypoidal choroidal vasculopathy (PCV) and response of hemorrhagic and exudative PCV to high-dose antiangiogenic therapy (an American Ophthalmological Society thesis). Trans Am Ophthalmol Soc. 2014;112:74-93.

24. Kawamura A, Yuzawa M, Mori R, Haruyama M, Tanaka K. Indocyanine green angiographic and optical coherence tomographic findings support classification of polypoidal choroidal vasculopathy into two types. Acta Ophthalmol. 2013;91: e474-481.

25. Tamachi T, Kohno T, Yamamoto M, Hirayama K, Kyo A, Ueda N, et al. One-Year Results of a Treat-and-Extend Regimen of Intravitreal Aflibercept for Polypoidal Choroidal Vasculopathy. Ophthalmol Ther. 2020;9:1069-82.

26. Rouvas A, Gouliopoulos N, Douvali M, Koutsocheras G, Theodorou M, Bouratzis N, et al. One year outcomes of treat and extend and pro re nata (PRN) treatment regimens with aflibercept for polypoidal choroidal vasculopathy. Eur J Ophthalmol. 2021. https://doi.org/10.1177/11206721211014717.

27. Hosokawa M, Morizane Y, Hirano M, Kimura S, Kumase F, Shiode Y, et al. One-year outcomes of a treat-and-extend regimen of intravitreal aflibercept for polypoidal choroidal vasculopathy. Jpn J Ophthalmol. 2017;61:150-8.

28. Teo KYC, Jordan-Yu JM, Tan ACS, Yeo IYS, Mathur R, Chan CM, et al. Efficacy of a novel personalised aflibercept monotherapy regimen based on polypoidal lesion closure in participants with polypoidal choroidal vasculopathy. $\mathrm{Br} \mathrm{J}$ Ophthalmol. 2021. https://doi.org/10.1136/bjophthalmol-2020-318354.

29. Freund KB, Korobelnik JF, Devenyi R, Framme C, Galic J, Herbert E, et al. Treatand-extend regimens with anti-vegf agents in retinal diseases: a literature review and consensus recommendations. Retina. 2015;35:1489-506.

30. Wykoff CC, Croft DE, Brown DM, Wang R, Payne JF, Clark L, et al. Prospective Trial of Treat-and-Extend versus Monthly Dosing for Neovascular Age-Related Macular Degeneration: TREX-AMD 1-Year Results. Ophthalmology. 2015;122:2514-22.

31. Ohji M, Takahashi K, Okada AA, Kobayashi M, Matsuda Y, Terano Y. Efficacy and Safety of Intravitreal Aflibercept Treat-and-Extend Regimens in Exudative AgeRelated Macular Degeneration: 52- and 96-Week Findings from ALTAIR: a randomized controlled trial. Adv Ther. 2020;37:1173-87.

32. Guymer RH, Markey CM, McAllister IL, Gillies MC, Hunyor AP, Arnold J, et al. Tolerating Subretinal Fluid in Neovascular Age-Related Macular Degeneration Treated with Ranibizumab Using a Treat-and-Extend Regimen: FLUID Study 24Month Results. Ophthalmology. 2019;126:723-34.

33. Evans RN, Reeves BC, Maguire MG, Martin DF, Muldrew A, Peto T, et al. Associations of Variation in Retinal Thickness With Visual Acuity and Anatomic Outcomes in Eyes With Neovascular Age-Related Macular Degeneration Lesions Treated With Anti-Vascular Endothelial Growth Factor Agents. JAMA Ophthalmol. 2020;138:1043-51.

34. Chakravarthy U, Havilio M, Syntosi A, Pillai N, Wilkes E, Benyamini G, et al. Impact of macular fluid volume fluctuations on visual acuity during anti-VEGF therapy in eyes with nAMD. Eye (Lond). 2021. https://doi.org/10.1038/s41433-020-01354-4.

35. Cheong KX, Teo AWJ, Cheung CMG, Too IHK, Chakravarthy U, Teo KYC. Association between retinal thickness variation and visual acuity change in neovascular age-related macular degeneration. Clin Exp Ophthalmol. 2021. https://doi. org/10.1111/ceo.13927.

36. Chaikitmongkol V, Upaphong P, Patikulsila D, Jirarattanasopa P, Choovuthayakorn J, Watanachai N, et al. Timing of Complete Polypoidal Regression Following Intravitreous Aflibercept Treatments in Polypoidal Choroidal Vasculopathy. Ophthalmol Retina. 2021. https://doi.org/10.1016/j.oret.2021.03.012.
37. Teo KYC, Jordan-Yu JM, Tan AC, Yeo IY, Mathur R, Chan CM, et al. Efficacy of a novel personalised aflibercept monotherapy regimen based on polypoidal lesion closure in participants with polypoidal choroidal vasculopathy. $\mathrm{Br} \mathrm{J}$ Ophthalmol. 2021. https://doi.org/10.1136/bjophthalmol-2020-318354.

38. Gomi F, Oshima Y, Mori R, Kano M, Saito M, Yamashita A, et al. Initial versus delayed photodynamic therapy in combination with ranibizumab for treatment of polypoidal choroidal vasculopathy: the Fujisan study. Retina. 2015; 35:1569-76.

39. Teo KYC, Sadda SR, Cheung CMG, Chakravarthy U, Staurenghi G, Invernizzi A, et al. Non ICGA treatment criteria for Suboptimal Anti VEGF Response for Polypoidal Choroidal Vasculopathy: APOIS PCV Workgroup Report 2. Ophthalmol Retina. 2021. https://doi.org/10.1016/j.oret.2021.04.002.

40. Chong Teo KY, Sadda SR, Gemmy Cheung CM, Chakravarthy U, Staurenghi G, Invernizzi A, et al. Non ICGA treatment criteria for Suboptimal Anti VEGF Response for Polypoidal Choroidal Vasculopathy: APOIS PCV Workgroup Report 2. Ophthalmol Retina. 2021. https://doi.org/10.1016/j.oret.2021.04.002.

41. Kokame GT, Omizo JN, Kokame KA, Yamane ML. Differentiating Exudative Macular Degeneration and Polypoidal Choroidal Vasculopathy Using Optical Coherence Tomography B-scan. Ophthalmol Retina. 2021. https://doi.org/ 10.1016/j.oret.2021.05.009.

42. Cheung CMG, Yanagi Y, Mohla A, Lee SY, Mathur R, Chan CM, et al. Characterization and differentiation of polypoidal choroidal vasculopathy using swept source optical coherence tomography angiography. Retina. 2017;37:1464-74.

43. Srour M, Querques G, Semoun O, El Ameen A, Miere A, Sikorav A, et al. Optical coherence tomography angiography characteristics of polypoidal choroidal vasculopathy. Br J Ophthalmol. 2016;100:1489-93.

44. Bo Q, Yan Q, Shen M, Song M, Sun M, Yu Y, et al. Appearance of Polypoidal Lesions in Patients With Polypoidal Choroidal Vasculopathy Using SweptSource Optical Coherence Tomographic Angiography. JAMA Ophthalmol. 2019; 137:642-50.

45. Kokame GT, Yeung L, Teramoto K, Lai JC, Wee R. Polypoidal choroidal vasculopathy exudation and hemorrhage: results of monthly ranibizumab therapy at one year. Ophthalmologica. 2014;231:94-102.

46. Warrow DJ, Hoang QV, Freund KB. Pachychoroid pigment epitheliopathy. Retina. 2013;33:1659-72.

47. Cheung CMG, Lee WK, Koizumi H, Dansingani K, Lai TYY, Freund KB. Pachychoroid disease. Eye (Lond). 2019;33:14-33.

48. Kim H, Lee SC, Kwon KY, Lee JH, Koh HJ, Byeon SH, et al. Subfoveal choroidal thickness as a predictor of treatment response to anti-vascular endothelial growth factor therapy for polypoidal choroidal vasculopathy. Graefes Arch Clin Exp Ophthalmol. 2016;254:1497-503.

49. Chang YC, Cheng CK. Difference between pachychoroid and nonpachychoroid polypoidal choroidal vasculopathy and their response to anti-vascular endothelial growth factor therapy. Retina. 2020;40:1403-11.

50. Jordan-Yu JM, Teo KYC, Chakravarthy U, Gan A, Tan ACS, Cheong KX, et al. Polypoidal choroidal vasculopathy features vary according to subfoveal choroidal thickness. Retina. 2021;41:1084-93.

51. Cheung CMG, Tan CS, Patalauskaite R, Margaron $P$, Lai TYY. Ranibizumab with or without verteporfin photodynamic therapy for polypoidal choroidal vasculopathy: predictors of Visual and Anatomical Response in the EVEREST II Study. Retina. 2021;41:387-92.

52. Yanagi Y, Ting DSW, Ng WY, Lee SY, Mathur R, Chan CM, et al. Choroidal vascular hyperpermeability as a predictor of treatment response for polypoidal choroidal vasculopathy. Retina. 2018;38:1509-17.

53. Koizumi H, Yamagishi T, Yamazaki T, Kinoshita S. Relationship between clinical characteristics of polypoidal choroidal vasculopathy and choroidal vascular hyperpermeability. Am J Ophthalmol. 2013;155:305-13.e301.

54. Sonoda S, Sakamoto T, Otsuka H, Yoshinaga N, Yamashita T, Ki IY, et al. Responsiveness of eyes with polypoidal choroidal vasculopathy with choroidal hyperpermeability to intravitreal ranibizumab. BMC Ophthalmol. 2013;13:43.

55. Cheong KX, Teo KYC, Cheung CMG. Influence of pigment epithelial detachment on visual acuity in neovascular age-related macular degeneration. Surv Ophthalmol. 2021;66:68-97.

56. Cheong KX, Grewal DS, Teo KYC, Gan ATL, Jaffe GJ, Cheung GCM. The relationship between pigment epithelial detachment and visual outcome in neovascular agerelated macular degeneration and polypoidal choroidal vasculopathy. Eye (Lond). 2020;34:2257-63.

57. Keenan TDL, Chakravarthy U, Loewenstein A, Chew EY, Schmidt-Erfurth U. Automated Quantitative Assessment of Retinal Fluid Volumes as Important Biomarkers in Neovascular Age-Related Macular Degeneration. Am J Ophthalmol. 2021;224:267-81.

58. You QS, Tsuboi K, Guo Y, Wang J, Flaxel CJ, Bailey ST, et al. Comparison of Central Macular Fluid Volume With Central Subfield Thickness in Patients With Diabetic Macular Edema Using Optical Coherence Tomography Angiography. JAMA Ophthalmol. 2021. https://doi.org/10.1001/jamaophthalmol.2021.1275. 
59. Dugel PU, Koh A, Ogura Y, Jaffe GJ, Schmidt-Erfurth U, Brown DM, et al. HAWK and HARRIER: Phase 3, Multicenter, Randomized, Double-Masked Trials of Brolucizumab for Neovascular Age-Related Macular Degeneration. Ophthalmology. 2020;127:72-84.

60. Dugel PU, Singh RP, Koh A, Ogura Y, Weissgerber G, Gedif K, et al. HAWK and HARRIER: Ninety-Six-Week Outcomes from the Phase 3 Trials of Brolucizumab for Neovascular Age-Related Macular Degeneration. Ophthalmology. 2021;128:89-99.

61. Jaffe GJ. Comparison of the Efficacy and Safety of Brolucizumab versus Aflibercept in eyes with Polypoidal Choroidal Vasculopathy: 96-Week Results from the HAWK Study. The American Academy of Ophthalmology Meeting, San Francisco, 2019.

62. Matsumoto H, Hoshino J, Mukai R, Nakamura K, Akiyama H. Short-term outcomes of intravitreal brolucizumab for treatment-naïve neovascular age-related macular degeneration with type 1 choroidal neovascularization including polypoidal choroidal vasculopathy. Sci Rep. 2021;11:6759.

63. Baumal CR, Spaide RF, Vajzovic L, Freund KB, Walter SD, John V, et al. Retinal Vasculitis and Intraocular Inflammation after Intravitreal Injection of Brolucizumab. Ophthalmology. 2020;127:1345-59.

64. Joussen AM, Ricci F, Paris LP, Korn C, Quezada-Ruiz C, Zarbin M. Angiopoietin/ Tie2 signalling and its role in retinal and choroidal vascular diseases: a review of preclinical data. Eye (Lond). 2021;35:1305-16.

65. Heier JS, Singh RP, Wykoff CC, Csaky KG, Lai TYY, Loewenstein A, et al. The angiopoietin/tie pathway in retinal vascular diseases: a review. Retina. 2021;41:1-19.

66. Roche. New phase III data show Roche's faricimab is the first investigational injectable eye medicine to extend time between treatments up to four months in two leading causes of vision loss, potentially reducing treatment burden for patients. Roche. https://www.roche.com/media/releases/med-cor-2021-02-12.htm. Accessed 18th May, 2021.

67. Campochiaro PA, Marcus DM, Awh CC, Regillo C, Adamis AP, Bantseev V, et al. The Port Delivery System with Ranibizumab for Neovascular Age-Related Macular Degeneration: Results from the Randomized Phase 2 Ladder Clinical Trial. Ophthalmology. 2019;126:1141-54.

68. Kim JH, Kim JY, Lee DW, Kim CG, Kim JW. Fibrovascular pigment epithelial detachment in eyes with subretinal hemorrhage secondary to neovascular AMD or PCV: a morphologic predictor associated with poor treatment outcomes. Sci Rep. 2020;10:14943.

69. Cheung CMG, Grewal DS, Teo KYC, Gan A, Mohla A, Chakravarthy U, et al. The Evolution of Fibrosis and Atrophy and Their Relationship with Visual Outcomes in Asian Persons with Neovascular Age-Related Macular Degeneration. Ophthalmol Retin. 2019;3:1045-55.

70. Chang YS, Kim JH, Kim JW, Kim CG, Lee DW. Development of Submacular Hemorrhage in Neovascular Age-related Macular Degeneration: influence on Visual Prognosis in a Clinical Setting. Korean J Ophthalmol. 2018;32:361-8.

71. Daniel E, Pan W, Ying GS, Kim BJ, Grunwald JE, Ferris FL 3rd, et al. Development and Course of Scars in the Comparison of Age-Related Macular Degeneration Treatments Trials. Ophthalmology. 2018;125:1037-46.

72. Sharma S, Toth CA, Daniel E, Grunwald JE, Maguire MG, Ying GS, et al. Macular Morphology and Visual Acuity in the Second Year of the Comparison of Age-Related Macular Degeneration Treatments Trials. Ophthalmology. 2016; 123:865-75.

73. Grunwald JE, Daniel E, Huang J, Ying GS, Maguire MG, Toth CA, et al. Risk of geographic atrophy in the comparison of age-related macular degeneration treatments trials. Ophthalmology. 2014;121:150-61.

74. Grunwald JE, Pistilli M, Ying GS, Maguire MG, Daniel E, Martin DF, et al. Growth of geographic atrophy in the comparison of age-related macular degeneration treatments trials. Ophthalmology. 2015;122:809-16.

75. Teo KYC, Joe AW, Nguyen V, Invernizzi A, Arnold JJ, Barthelmes D, et al. Prevalence and Risk Factors for the Development of Physician-Graded Subretinal Fibrosis in Eyes Treated for Neovascular Age-Related Macular Degeneration. Retina. 2020;40:2285-95.

76. Gillies M, Arnold J, Bhandari S, Essex RW, Young S, Squirrell D, et al. Ten-Year Treatment Outcomes of Neovascular Age-Related Macular Degeneration from Two Regions. Am J Ophthalmol. 2020;210:116-24.

77. Wolff B, Macioce V, Vasseur V, Castelnovo L, Michel G, Nguyen V, et al. Ten-year outcomes of anti-vascular endothelial growth factor treatment for neovascular age-related macular disease: a single-centre French study. Clin Exp Ophthalmol. 2020;48:636-43.

78. Kikushima W, Sakurada Y, Sugiyama A, Yoneyama S, Matsubara M, Fukuda Y, et al. Five-Year Outcome of Aflibercept Monotherapy for Exudative AgeRelated Macular Degeneration with Good Baseline Visual Acuity. J Clin Med. 2021;10:1098.

79. Wataru K, Sugiyama A, Yoneyama S, Matsubara M, Fukuda Y, Parikh R, et al. Fiveyear outcomes of photodynamic therapy combined with intravitreal injection of ranibizumab or aflibercept for polypoidal choroidal vasculopathy. PLoS ONE. 2020;15:e0229231.

80. Kim JH, Chang YS, Kim JW, Kim CG, Lee DW. Submacular hemorrhage and grapelike polyp clusters: factors associated with reactivation of the lesion in polypoidal choroidal vasculopathy. Eye (Lond). 2017;31:1678-84.

81. Cho JH, Ryoo NK, Cho KH, Park SJ, Park KH, Woo SJ. Incidence Rate of Massive Submacular Hemorrhage and its Risk Factors in Polypoidal Choroidal Vasculopathy. Am J Ophthalmol. 2016;169:79-88.

82. Cho SC, Cho J, Park KH, Woo SJ. Massive submacular haemorrhage in polypoidal choroidal vasculopathy versus typical neovascular age-related macular degeneration. Acta Ophthalmol. 2020. https://doi.org/10.1111/aos.14676.

83. Azuma K, Okubo A, Nomura Y, Zhou H, Terao R, Hashimoto $Y$, et al. Association between pachychoroid and long-term treatment outcomes of photodynamic therapy with intravitreal ranibizumab for polypoidal choroidal vasculopathy. Sci Rep. 2020;10:8337.

84. Baek J, Lee JH, Jeon S, Lee WK. Choroidal morphology and short-term outcomes of combination photodynamic therapy in polypoidal choroidal vasculopathy. Eye. 2019;33:419-27.

85. Maruko I, lida T, Sugano Y, Saito M, Sekiryu T. Subfoveal retinal and choroidal thickness after verteporfin photodynamic therapy for polypoidal choroidal vasculopathy. Am J Ophthalmol. 2011;151:594-603 e591.

86. Teo KYC, Yanagi Y, Lee SY, Yeo IYS, Tan GSW, Mathur R, et al. Comparison of Optical Coherence Tomography Angiographic Changes after Anti-Vascular Endothelial Growth Factor Therapy Alone or in Combination with Photodynamic Therapy in Polypoidal Choroidal Vasculopathy. Retina. 2018;38: 1675-87.

87. Gemmy Cheung CM, Chong Teo KY, Spaide R. Pulsatile Filling of Dilated Choroidal Vessels in Macular Watershed Zones. Retina. 2021. https://doi.org/ 10.1097/IAE.0000000000003195.

88. Spaide RF, Ledesma-Gil G, Gemmy Cheung CM. Intervortex venous anastomosis in Pachychoroid-related disorders. Retina. 2021;41:997-1004.

89. Okamoto N, Tobe T, Hackett SF, Ozaki H, Vinores MA, LaRochelle W, et al. Transgenic mice with increased expression of vascular endothelial growth factor in the retina: a new model of intraretinal and subretinal neovascularization. Am J Pathol. 1997;151:281-91.

90. Jones A, Kumar S, Zhang N, Tong Z, Yang JH, Watt C, et al. Increased expression of multifunctional serine protease, HTRA1, in retinal pigment epithelium induces polypoidal choroidal vasculopathy in mice. Proc Natl Acad Sci USA. 2011;108:14578-83.

91. Kumar S, Berriochoa Z, Ambati BK, Fu Y. Angiographic features of transgenic mice with increased expression of human serine protease HTRA1 in retinal pigment epithelium. Investig Ophthalmol Vis Sci. 2014;55:3842-50.

92. Li M, Zhang X, Liao N, Ye B, Peng Y, Ji Y, et al. Analysis of the Serum Lipid Profile in Polypoidal Choroidal Vasculopathy. Sci Rep. 2016;6:38342.

93. Xu N, Xu H, Zhao M, Xu Y, Huang L. Associations of systemic, serum lipid and lipoprotein metabolic pathway gene variations with polypoidal choroidal vasculopathy in China. PLoS ONE. 2019;14:e0226763.

94. Liu K, Chen LJ, Lai TY, Tam PO, Ho M, Chiang SW, et al. Genes in the high-density lipoprotein metabolic pathway in age-related macular degeneration and polypoidal choroidal vasculopathy. Ophthalmology. 2014;121:911-6.

95. Deng Y, Shuai P, Wang H, Zhang S, Li J, Du M, et al. Untargeted metabolomics for uncovering plasma biological markers of wet age-related macular degeneration. Aging (Albany NY). 2021;13:13968-14000.

96. Lu Z, Lin V, May A, Che B, Xiao X, Shaw DH, et al. HTRA1 synergizes with oxidized phospholipids in promoting inflammation and macrophage infiltration essential for ocular VEGF expression. PLoS ONE. 2019;14:e0216808.

97. Tom I, Pham VC, Katschke KJ Jr., Li W, Liang WC, Gutierrez J, et al. Development of a therapeutic anti-HtrA1 antibody and the identification of DKK3 as a pharmacodynamic biomarker in geographic atrophy. Proc Natl Acad Sci USA. 2020;117:9952-63.

98. Teo KYC, Gillies M, Fraser-Bell S. The Use of Vascular Endothelial Growth Factor Inhibitors and Complementary Treatment Options in Polypoidal Choroidal Vasculopathy: a Subtype of Neovascular Age-Related Macular Degeneration. Int J Mol Sci. 2018;19:2611.

99. Koh A, Lai TYY, Takahashi K, Wong TY, Chen LJ, Ruamviboonsuk P, et al. Efficacy and Safety of Ranibizumab With or Without Verteporfin Photodynamic Therapy for Polypoidal Choroidal Vasculopathy: a Randomized Clinical Trial. JAMA Ophthalmol. 2017;135:1206-13.

\section{AUTHOR CONTRIBUTIONS}

BJF, CMGC, and KYCT conceived and drafted the review outline. BJF wrote the paper. CMGC, SSS, WKL, GS, TYYL, PR, GK, YY, and KYCT provided critical review of the paper. 


\section{COMPETING INTERESTS}

The authors declare no competing interests.

\section{ADDITIONAL INFORMATION}

Correspondence and requests for materials should be addressed to K.Y.C.T.

Reprints and permission information is available at http://www.nature.com/ reprints

Publisher's note Springer Nature remains neutral with regard to jurisdictional claims in published maps and institutional affiliations.
Open Access This article is licensed under a Creative Commons Attribution 4.0 International License, which permits use, sharing, adaptation, distribution and reproduction in any medium or format, as long as you give appropriate credit to the original author(s) and the source, provide a link to the Creative Commons license, and indicate if changes were made. The images or other third party material in this article are included in the article's Creative Commons license, unless indicated otherwise in a credit line to the material. If material is not included in the article's Creative Commons license and your intended use is not permitted by statutory regulation or exceeds the permitted use, you will need to obtain permission directly from the copyright holder. To view a copy of this license, visit http://creativecommons.org/licenses/by/4.0/.

(c) The Author(s) 2021 